\title{
Body weight and self-perception are associated with depression: Results from the National Health and Nutrition Examination Survey (NHANES) 2005-2016
}

\author{
Darimont, Tobia ; Karavasiloglou, Nena ; Hysaj, Ola ; Richard, Aline ; Rohrmann, Sabine
}

\begin{abstract}
BACKGROUND Despite evidence suggesting that perceptual body image measurements are strongly associated with depression, few studies examined the association between perceptual body image and depression in adults. This study aimed to investigate the association of different measures of perceptual body image measurements with depression. METHODS We analyzed data of 22,735 adults participating in the National Health and Nutrition Examination Survey between 2005 and 2016. Depression was ascertained using the validated Patient Health Questionnaire (PHQ-9) and depression was defined as PHQ score 10. The associations of measured body mass index (BMI) and body image (i.e., self-reported BMI, BMI discordance, perceived weight, and desired weight) with depression were assessed using logistic regression. RESULTS Women perceiving themselves as overweight or reporting their BMI as obese had significantly higher odds of depression (odds ratio [OR] 1.48, 95\% confidence interval [CI] 1.17-1.72 and 1.29, 1.04-1.60, respectively) compared to women perceiving themselves as about the right weight or reporting their BMI as normal. The association between perceived overweight and depression was independent of measured BMI (OR 1.37, 95\% CI 1.09-1.72). Men perceiving themselves as underweight had higher odds of depression (OR 1.50, 95\% CI 1.06-2.11) than men perceiving themselves as about the right weight. In women, but not in men, measured obesity was associated with higher odds of depression. LIMITATIONS The study's cross-sectional design and missing information on comorbidities. CONCLUSION Weight perception (in women and men), measured BMI and BMI discordance in women are associated with depression..
\end{abstract}

DOI: https://doi.org/10.1016/j.jad.2020.05.130

Posted at the Zurich Open Repository and Archive, University of Zurich

ZORA URL: https://doi.org/10.5167/uzh-196202

Journal Article

Accepted Version

Originally published at:

Darimont, Tobia; Karavasiloglou, Nena; Hysaj, Ola; Richard, Aline; Rohrmann, Sabine (2020). Body weight and self-perception are associated with depression: Results from the National Health and Nutrition Examination Survey (NHANES) 2005-2016. Journal of Affective Disorders, 274:929-934.

DOI: https://doi.org/10.1016/j.jad.2020.05.130 
Body weight and self-perception are associated with depression. Results from the National Health and Nutrition Examination Survey (NHANES) 2005-2016

Short title: Body weight, self-perception linked to depression

Tobia Darimont ${ }^{1}$, Nena Karavasiloglou'1, Ola Hysaj', Aline Richard ${ }^{1}$, Sabine Rohrmann ${ }^{1}$

\section{Affiliations}

1 Division of Chronic Disease Epidemiology, Epidemiology, Biostatistics and Prevention Institute, University of Zurich, Zurich, Switzerland

\section{Corresponding Author}

Sabine Rohrmann

Division of Chronic Disease Epidemiology

Epidemiology, Biostatistics and Prevention Institute (EBPI)

University of Zurich

8002 Zurich

Switzerland

e-mail address: sabine.rohrmann@uzh.ch

phone: +41446345256 


\begin{abstract}
Background: Despite evidence suggesting that perceptual body image measurements are strongly associated with depression, few studies examined the association between perceptual body image and depression in adults. This study aimed was to investigate the association of between different measures of perceptual body image measurements with depression.
\end{abstract}

Methods: We analyzed data of 22,735 adults participating in the National Health and Nutrition Examination Survey between 2005 and 2016. Depression was ascertained using the validated Patient Health Questionnaire (PHQ-9) and depression was defined as PHQ score $\geq 10$. The associations of measured body mass index (BMI) and body image (i.e., self-reported BMI, BMI discordance, perceived weight, and desired weight) with depression were assessed using logistic regression.

Results: Women perceiving themselves as overweight or reporting their BMI as obese had significantly higher odds of depression (odds ratio [OR] 1.48, 95\% confidence interval [Cl] 1.17-1.72 and 1.29, 1.04-1.60, respectively) compared to women perceiving themselves as about the right weight or reporting their BMI as normal. The association between perceived overweight and depression was independent of measured BMI (OR 1.37, 95\% CI 1.09-1.72). Men perceiving themselves as underweight had higher odds of depression (OR 1.50, 95\% $\mathrm{Cl} 1.06-2.11$ ) than men perceiving themselves as about the right weight. In women, but not in men, measured obesity was associated with higher odds of depression.

Limitations: The study's cross-sectional design and missing information on comorbidities.

Conclusion: Weight perception (in women and men), measured BMI and BMI discordance in women are associated with depression.

Keywords: clinically relevant depression, weight perception, obesity, overweight, NHANES 


\section{Introduction}

According to the World Health Organization, depression is the leading cause of disability around the world and the fourth leading contributor to the global burden of disease (World Health Organisation, 2019). Given the detrimental effects of depression on physical and mental well-being (Moussavi et al., 2007), it is crucial to explore potentially modifiable factors that could influence depression in order to improve clinical care and prevention strategies.

Body Mass Index (BMI) has been strongly associated with depression consistently (de Wit et al., 2009; Luppino et al., 2010). A Dutch longitudinal study of 43,534 participants discovered a U-shaped relation between BMI and depression (de Wit et al., 2009); further evidence suggests a bidirectional relationship between depression and obesity (Mannan et al., 2016). Emerging evidence suggests that body image is another factor that is strongly associated with depression; it may even have a greater effect on depression than actual BMI or weight status per se (Ali et al., 2010; Holsen et al., 2001; Roberts and Duong, 2013).

Body image has been defined as a person's body-related self-perceptions and self-attitudes, including body-related thoughts, feelings, and behaviors (Cash, 2004; Cash, 1990; Slade, 1994). The concept of body image includes at least two components: perceptual body image size (i.e.,estimation of one's how people perceive the size, weight, and shape of their body) and attitudinal body image (affective, cognitive, and behavioral concerns with one's body size, weight, and shape) (Rucker III and Cash, 1992). Both, BMI and depression can influence the perception of body image in isolation but also in combination (Paans et al., 2018). Hence, Friedman and Bownell proposed body image as a potential mediator of the relationship between BMI and depression (Friedman et al., 2002).

In the past decades, most research on the association between depression and perceptual body image has been focused on adolescents given the assumption that perceptual body image is affected by rapid pubertal development (Al Mamun et al., 2007; Holsen et al., 2001; Kamody et al., 2018; Lim and Kim, 2017; Roberts and Duong, 2013; Smolak, 2004). However, the physical changes that occur in adulthood can also affect body image (Halliwell and Dittmar, 2003). In this stage, body shape can undergo dramatic changes moving away from the common aesthetics of sociocultural body image ideals such as the extremely thin female body ideal and the muscular male body ideal (conveyed especially in western countries through the mass media) (Dittmar and Howard, 2004; Halliwell and Dittmar, 2003; Mintem et al., 2015). Some studies have explored associations between other perceptual body image measurements such as perceived weight or perceived BMI and depression in adults (Gaskin et al., 2013; Hong and Hong, 2019; Jackson et al., 2014; Kim et al., 2018; Lim and Kim, 2017; Paans et al., 2018; Richard et al., 2016; Thurston et al., 2017). However, these investigations have been either studies based on specific subgroup populations, i.e., Asian populations (Hong and Hong, 2019; Kim et al., 2018; Lim and Kim, 2017), middle-aged women (Jackson et al., 2014), overweight youth (Thurston et al., 2017), or have been based on small sample populations (total recruited participants 1,452) (Paans et al., 2018). 
In a previous analysis of the National Health and Nutrition Examination Survey (NHANES), which included cycles 2005-2006 and 2007-2008, Gaskin et al. showed that adult women who perceived themselves as underweight or overweight were more likely to be depressed than women who perceived themselves to be about the right weight. This associations was independent of their actual (i.e., measured) weight (Gaskin et al., 2013). Among men, perceiving oneself as underweight was associated with increased odds of depression. However, this study only considered some of the potential confounders (race/ethnicity, age, family income, education, smoking, and alcohol consumption). Other related confounders such as physical activity, self-perceived health or marital status, which have been associated with depression and body image (LaPierre, 2009; Nguyen et al., 2017; Takacs, 2014), were not considered by Gaskin and colleagues (Gaskin et al., 2013).

Thus, given the scant research on depression and body image perception conducted in adults we aimed to extend the existing literature by assessing the association of measured BMI and perceptual body image measurements such as self-reported BMI, BMI discordance, perceived weight, and weight dissatisfaction with depression. To this end, we used data from NHANES, a nationwide populationbased survey, conducted in the United States.

\section{Methods}

\section{Population}

Data from NHANES between 2005 and 2016 were used in our analyses. NHANES is a nationwide population-based survey, conducted in the United States. It collects nationally representative data on health and nutrition, using a complex, multistage probability sample of the US civilian and noninstitutionalized population (Borrud et al., 2014; Zipf et al., 2013). NHANES participants are interviewed and undergo physical examinations in a mobile examination center. NHANES data are publically available and can be accessed online (http://www.cdc.gov).

The total sample size of the NHANES 2005-2016 was 60,936. In this analysis, only participants older than 20 years who gave a valid answer to all questions of the Patient Health Questionnaire (PHQ-9) (Kroenke et al., 2001) were included, since not all standard adult measurements and in particular alcohol consumption were assessed for these participants. Participants younger than 20 years old were excluded ( $n=26,756$ ). Participants whose answer was "Refused" or "Don't know" or with a missing answer in at least one of the PHQ questions were excluded from the analyses $(n=4,867)$. Also, participants with missing information or those who replied "Refused" or "Don't know" in any of the body image components were excluded from the analyses $(n=1,219)$. Participants who used a proxy to fill out the questionnaire or had missing information regarding proxy usage were also excluded $(n=47)$ as well as participants who answered "Refused" or "Don't know" or had missing information regarding their physical activity or alcohol consumption $(n=5,300)$. Finally, participants with missing information on any of the remaining confounding variables were excluded $(n=12)$. The final study population included 22,735 participants. 


\section{Measured Body Mass Index and Perceptual body image measurements}

To assess the possible association of measured BMI and body image with depression, we used measured BMI and four different weight-related perceptual body image measurements (self-reported BMI, BMI discordance, current weight perception and desired weight), as presented below.

Trained health technicians, accompanied by a recorder, performed the data collection of all body measurements. Measured BMI was reported in the NHANES as the fraction of weight (in kg)/ height ${ }^{2}$ (in meters). We estimated the self-reported BMI for each participant using its self-reported body weight (in pounds) and the self-reported height (in inches). After converting the weight and height to the metric scale, self-reported BMI was calculated as weight (in $\mathrm{kg}$ )/ height ${ }^{2}$ (in meters). Both measured and self-reported BMI were categorized as underweight $\left(B M I<18,5 \mathrm{~kg} / \mathrm{m}^{2}\right)$, normal weight (BMI 18.5$24.9 \mathrm{~kg} / \mathrm{m}^{2}$ ), overweight (BMI $25.0-29.9 \mathrm{~kg} / \mathrm{m}^{2}$ ), or obese $\left(\mathrm{BMI} \geq 30 \mathrm{~kg} / \mathrm{m}^{2}\right.$ ) according to the World Health Organization BMI classification (World Health Organization, 2000).

We compared the concordance between measured and self-reported BMI categories (hereafter called BMI discordance). Participants were classified into three groups: a) concordance, if their self-reported $\mathrm{BMI}$ and their measured BMI belonged in the same BMI category, b) over-estimation, if self-reported $\mathrm{BMI}$ belonged to a higher BMI category than measured BMI and c) under-estimation, if self-reported $\mathrm{BMI}$ belonged to a lower BMI category than measured BMI.

Current weight perception was defined as "overweight", "underweight", or "about the right weight" based on the answer to the question "Do you consider yourself to be...?" Desired weight was classified as "more", "less" or "stay about the same" based on the answer to the question "Would you like to weigh...?" Both questions were asked during the interview, using an interviewer-administered Computer-Assisted Personal Interviewing (CAPI) system.

\section{Assessment of Depression}

The outcome of interest was depression and was assessed with the Patient Health Questionnaire (PHQ-9). This short screening questionnaire is a valid tool to assess depression by scoring each of the 9 Diagnostic Statistical Manual of Mental Disorders signs and symptoms for depression from "0" (not at all) to "3" (nearly every day) (Kroenke et al., 2001). The sum of all answers to the PHQ questions is the PHQ score for each participant (range 0-27). The PHQ-9 is a valid DSM-V-based criteria instrument. From a possible total score of 27 , the cut-off point of $\geq 10$ has shown a sensitivity of $88 \%$ and a specificity of $88 \%$ for the diagnosis of major depression (Kroenke et al., 2001). Thus, we dichotomized a participant's PHQ-9 score into $<10$ (no depression) and $\geq 10$ (depression).

The main difference between our study and other studies published in the literature was the selection of the cut-off point in the PHQ score. While we have used the cut-off point of $\geq 10$, as described above, other studies have decided to use lower (Kim et al., 2018) or higher (Gaskin et al., 2013) cut-off points to define depression. 


\section{Covariates}

Information on age at study entry, race/ethnicity, education, and marital status was obtained via the demographics questionnaires. Trained interviewers using the CAPI system asked the demographics questionnaires at home.

Information on smoking was obtained for adults 20 years and older during the home interview by trained interviewers using the CAPI system. Participants responded whether they had smoked at least 100 cigarettes in their lifetime; and whether they currently smoked cigarettes (daily, some days, or not at all). Participants were categorized as never-smokers ( $<100$ cigarettes over the lifetime), former smokers (having smoked $>100$ cigarettes over the lifetime but do not currently smoke), and current smokers.

The physical activity questionnaire was based on the Global Physical Activity Questionnaire and included questions related to daily activities, leisure time activities, and sedentary activities. Physical activity was assessed during the home interview using the CAPI system. Participants were asked whether they participate in activities of moderate or vigorous intensity, outside of work or transportation, with a duration of at least 10 continuous minutes. Participants who answered "Yes" were classified as physically active, whereas those who answered "No" were classified as nonphysically active, similar to as in a previous study by Vásquez et al (Vásquez et al., 2014).

Information regarding self-rated health status was obtained during the physical exam, at the examination center, using the CAPI system. Alcohol consumption was assessed in adults 20 years and older during the physical exam, at the examination center, using the CAPI system. Participants were classified in sex-specific categories based on their self-reported average daily alcohol consumption: non-drinker, moderate drinker ( $\leq 1$ alcoholic drink per day), binge drinker ( $>1$ and $<4$ alcoholic drinks per day) or heavy drinker ( $\geq 4$ alcoholic drinks per day) for women; non-drinker, moderate drinker ( $\leq 2$ alcoholic drink per day), binge drinker ( $>2$ and $<5$ alcoholic drinks per day) or heavy drinker ( $\geq 5$ alcoholic drinks per day) for men, similar as in a previous analysis by Agrawal et al (Agrawal et al., 2018).

\section{Statistical analysis}

Baseline categorical data were expressed as percentages and continuous data as means and standard errors (SE). Logistic regression models (using the PROC SURVEYLOGISTIC function in SAS) were used to determine the association between the selected body image components and the odds of depression. Since considerable differences in the association between body image components and the odds of depression were detected between sexes, the results were reported separately for men and women. We built three models in sequence as follows. The first model (Model 1) was adjusted only for age at study entry (years), and race/ethnicity (non-Hispanic white, nonHispanic black, Mexican American, other). The second model (Model 2) additionally adjusted for several a-priori determined confounders based on the existing literature including smoking (never, former, current smoker), education (high school or less, some college or more, unknown) (Loprinzi and 
Loenneke, 2018), marital status (never married/widowed, divorced/separated, married/living as married, unknown) (Goldfarb-Rumyantzev et al., 2010), physical activity (active versus inactive), alcohol consumption (non-drinker, moderate drinker, binge drinker, heavy drinker) and self-rated health status (excellent, very good, good, fair, poor, don't know). The third model (Model 3) was additionally adjusted for measured BMI (continuous), where appropriate. The results were presented as odds ratios (OR) and corresponding $95 \%$ confidence intervals $(\mathrm{Cl})$.

All statistical analyses were performed using SAS version 9.4 (SAS Institute, Cary, NC) and significance levels were set at alpha $=0.05$. Sampling weights adapted to the inclusion of multiple NHANES cycles were used in all analyses to account for the complex survey design and survey nonresponse.

\section{Results}

This study included 22,735 participants (10,887 women and 11,848 men; unweighted counts). Most study participants were non-Hispanic white and almost two-thirds had attended at least some college (65.8\% women, $60.3 \%$ men; Table 1$)$. More than half of the study participants were non-smokers ( $56.0 \%$ women, $46.3 \%$ men). Nearly $40 \%$ of either sex ( $43.6 \%$ women, $40.2 \%$ men) reported being to be physically inactive. Almost one third of women and nearly $40 \%$ of men were overweight and just under $40 \%$ in both sexes were obese (37.7\% women, $36.0 \%$ men). Overall, $7.9 \%$ of women and $4.5 \%$ of men reported depression.

When adjusting only for race/ethnicity and age (Model 1), being classified as overweight or obese according to measured or self-reported BMI, compared to being classified as normal weight, was associated with higher likelihood of depression in women (Table 2). Additionally, women who perceived themselves as overweight or underweight and those wanting to either lose or gain weight had higher odds of depression, compared to women themselves as being "about the right weight", or those wanting to stay at the same weight, respectively. Men classified as overweight according to their measured BMI had lower odds of depression compared to men classified as normal weight.

Furthermore, men who perceived themselves as underweight, as well as those wanting to weigh more, had higher likelihood of depression than men who perceived themselves as having "about the right weight", or those wanting to stay at the same weight, respectively.

When taking into account additional confounders, several results were attenuated (Model 2). In women, obesity (for both, measured and self-reported BMI) was associated with an increased likelihood of depression, compared to normal weight. Furthermore, women who perceived themselves as overweight had higher odds of depression (OR 1.48, 95\% Cl 1.17-1.72), compared to those who perceived themselves as being "about the right weight". On the other hand, women who overestimated their BMI category had lower odds of depression compared to women who correctly estimated their BMI category (OR $0.62,95 \% \mathrm{Cl} 0.40-0.95)$. In men, only the association between depression and perceiving themselves as underweight vs. "about the right weight" (OR 1.50, 95\% $\mathrm{Cl} 1.06-2.11$ ) remained statistically significant. 
When further adjusting for measured BMI, the association between women who perceived themselves as overweight and depression remained consistent but was slightly attenuated (OR 1.37, 95\% Cl 1.091.72). A similar attenuation was observed in men who perceived themselves weight as underweight, where the association lost statistical significance (OR 1.42, 95\% Cl 0.99-2.02).

\section{Discussion}

Our results add to the current knowledge on the association between weight perception, self-reported $\mathrm{BMI}$, measured BMI and depression. Our findings suggest that women whose self-reported body weight and height classified them as obese or women who perceived themselves as overweight had higher odds of depression, whereas men who perceived themselves as underweight had higher odds of depression. Upon adjustment for measured BMI, only the results related to women's current weight perception remained significant. Lower odds of depressive symptoms were also observed for women whose self-reported BMI classified them in a higher BMI category compared to their measured BMI (BMI discordance).

Studies in the literature have reported an association between measured body weight and depression (Gray et al., 2012; Jackson et al., 2014). However, others suggest an association between perceived body weight and depression, but not between measured body weight and depression (Kim et al., 2018; Roberts and Duong, 2013; Tang et al., 2010). A previous analysis using NHANES data (cycles 2005-2006 and 2007-2008) observed significant associations between perceived overweight and depression in women as well as between perceived underweight and depression in men; statistically significant positive associations were reported for overweight and obese women (measured BMI), while no associations were observed between measured BMI and depression for men (Gaskin et al., 2013). Our findings on current weight perception and depression in both sexes, as well as those for obese women (measured BMI category) are in line with those of the previous NHANES analysis.

We detected an inverse, statistically significant association between the over-estimation of selfreported BMI and depression (BMI discordance; here self-reported BMI classifying participants in a higher BMI category compared to their measured BMI). Results from the Study of Women's Health Across the Nation (SWAN) suggested that women with body image discordance have an inverse, nonstatistically significant association with depressive symptoms compared to those with body image concordance (Jackson et al., 2014). Our results are in line with these of the SWAN. A possible explanation for our finding could be the phenomenon of "depressive realism". The "depressive realism hypothesis" supports that depressed individuals cannot only make realistic judgments but that they can do so to a greater extent than non-depressed individuals (Moore and Fresco, 2012). Thus, it is possible that the observed finding is a reflection of the realistic judgment of self-reported BMI compared to measured BMI (i.e. BMI concordance) in depressed individuals compared to the nonrealistic judgment (either as over- or under-estimation) of the non-depressed individuals. 
Our findings of significant associations between perceived overweight and depression in women and between perceived underweight with depression in men could be explained by sex-specific differences in body ideals. Generally, men may prefer a more muscular body, compared to the slender female body according to the western body ideals. Internalization of these body ideals can lead to body dissatisfaction (Frederick et al., 2007; Rodgers et al., 2015). Body image dissatisfaction has been associated with depression in previous studies (Silva et al., 2019). However, hormonal and physiological changes over the life course, developmental difficulties in childhood and puberty, pregnancy, social-comparison, and other psychosocial, sociocultural, biological and environmental factors might also explain why women are more frequently dissatisfied with their body weight and shape (Deeks, 2004; Sowers et al., 2007).

Health prevention programs should focus on obesity and depression. Perceived body image has been highlighted as an important factor (Silva et al., 2019), in addition to BMI, that needs to be addressed in such programs. Programs that aim to induce a change of thinking about the thin beauty body ideal may help reduce the incidence of depression. Additionally, societal efforts to reduce the weight/obesity stigma can potentially assist the reduction of mental health and eating disorders.

The current study has several strengths, especially the representative, large sample $(22,546$ participants). NHANES participants were selected from the general, non-institutionalized population, thus conclusions can be largely generalizable. An additional strength of the study is the use of the PHQ-9, which is a valid DSM-V based criteria instrument with high sensitivity and specificity (Spitzer et al., 2014), to identify study participants with depression. Finally, the detailed data collection in the NHANES allowed us to adjust our analyses for several important confounders that might influence depression.

Several aspects of our study merit further discussion. We used BMI (both measured and self-reported) to classify individuals. However, BMI is an imperfect measure of body fatness, since high BMI does not necessarily reflect high-fat mass, but it might also indicate high muscle mass (Evans et al., 2006). Another limitation is the lack of information regarding other psychiatric disorders, present or past medication use, weight control practices, family history of psychiatric disorders, or past cognitive behavior change practices. The study's cross-sectional design does not allow us to establish the timeline between body image components and depression and thus we cannot distinguish cause and effect. Further research on the topic, using longitudinal data is needed.

\section{Conclusion}

Our findings, using a representative sample of the US population, suggest that current weight perception (in both women and men), and measured BMI and BMI discordance in women are associated with depression. These results need to be confirmed and further explored in longitudinal studies. Programs that induce a change of thinking in weight perception, in particular with the background of the unhealthy widespread western thin body ideal among women, can be a useful, 
additional approach to the prevention of depression. However, thus far, perceived overweight and obesity are not fully acknowledged in health prevention programs.

Declarations of interest: none.

This research did not receive any specific grant from funding agencies in the public, commercial, or not-for-profit sectors. 
Table 1. Socioeconomic and lifestyle characteristics of the study population by sex, NHANES 20052016

Women $(n=10,887) \quad$ Men $(n=11,848)$

Age at study entry, years, mean (SE)

$47.0(0.3)$

$45.9(0.3)$

Race/ethnicity, \%

Non-Hispanic White

72.1

69.8

Non-Hispanic Black

11.1

9.9

Mexican American

6.5

8.7

Other

10.3

11.6

Citizenship Status, \%

U.S.

94.6

91.2

Non U.S.

5.4

Education ${ }^{1}, \%$

High-school or less

34.2

39.7

Some college or more

65.8

60.3

Marital status ${ }^{1}, \%$

Never married/Widowed

24.0

22.0

Divorced/Separated

15.8

10.4

Married/Living as married

60.2

67.6

Smoking, \%

Never smoker

56.0

46.3

Former smoker

23.3

29.4

Current smoker

20.6

24.2

Alcohol consumption, \%

Non drinker

18.9

17.5

Moderate drinker

36.1

43.9

Binge drinker

35.1

20.8

Heavy drinker

10.0

17.8

Self-rated health status, \%

Excellent

10.5

Very good

35.6

31.4

Good

37.4

40.8 
Physical activity, \%

Moderate and/or vigorous

Inactive

Body Mass Index, measured, \%

Underweight

Normal weight

Overweight

Obese

Body Mass Index, self-reported, \%

Underweight

1.9

Normal weight

37.0

Overweight

27.8

40.3

Obese

33.3

Current weight perception, \%

About the right weight

Overweight

64.1

50.3

Underweight

Desired Weight, \%

Stay about the same

20.5

More

Less

76.3

58.9

Clinically relevant depression (PHQ score $\geq 10$ ), \%

7.9 
Table 2. Sex-specific associations of measured BMI, self-reported BMI, BMI discordance, current weight perception, and desired weight with clinically relevant depression ${ }^{1}$, NHANES 2005-2016

Women $(n=10,887)$

Men $(n=11,848)$

\begin{tabular}{|c|c|c|c|c|c|c|}
\hline & Model $1^{2}$ & Model $2^{2}$ & Model $3^{2}$ & Model $1^{2}$ & Model $2^{2}$ & Model $3^{2}$ \\
\hline \multicolumn{7}{|l|}{ Self-reported BMI ${ }^{3}$ category } \\
\hline Normal weight $\left(18.5-24.9 \mathrm{~kg} / \mathrm{m}^{2}\right)$ & 1.00 & 1.00 & 1.00 & 1.00 & 1.00 & 1.00 \\
\hline Overweight (25.0-29.9 kg/m²) & $1.29(1.05-1.60)$ & $0.97(0.78-1.21)$ & $0.96(0.76-1.21)$ & $0.77(0.58-1.01)$ & $0.87(0.64-1.17)$ & $0.92(0.67-1.26)$ \\
\hline Obese $\left(>30 \mathrm{~kg} / \mathrm{m}^{2}\right)$ & $2.36(1.95-2.85)$ & $1.29(1.04-1.60)$ & $1.24(0.85-1.81)$ & $0.98(0.73-1.32)$ & $0.79(0.57-1.10)$ & $0.93(0.59-1.47)$ \\
\hline Underweight (< $\left.18.5 \mathrm{~kg} / \mathrm{m}^{2}\right)$ & $1.36(0.54-3.39)$ & $0.68(0.27-1.72)$ & $0.69(0.27-1.76)$ & $1.29(0.58-2.86)$ & $0.75(0.30-1.84)$ & $0.70(0.28-1.74)$ \\
\hline
\end{tabular}

\section{Measured BMI category}

Normal weight $\left(18.5-24.9 \mathrm{~kg} / \mathrm{m}^{2}\right)$

1.00

1.00

Overweight $\left(25.0-29.9 \mathrm{~kg} / \mathrm{m}^{2}\right)$

Obese $\left(>30 \mathrm{~kg} / \mathrm{m}^{2}\right)$

$1.36(1.07-1.72) \quad 1.07(0.83-1.37)$

$2.30(1.91-2.78) \quad 1.27(1.03-1.57)$

$1.11(0.48-2.55) \quad 0.44(0.19-1.03)$

Underweight $\left(<18.5 \mathrm{~kg} / \mathrm{m}^{2}\right)$

\section{BMI discordance}

Concordance (Correct)

1.00

1.00

Over-estimation

$0.82(0.55-1.22) \quad 0.62(0.40-0.95)$

Under-estimation
1.00

1.00

- $\quad 0.74(0.55-1.00) \quad 0.88(0.63-1.22)$

- $\quad 0.89(0.66-1.20) \quad 0.76(0.54-1.06)$

- $\quad 1.21(0.62-2.37) \quad 0.61(0.30-1.22)$

1.00

1.00

$1.40(0.85-2.31) \quad 1.12(0.65-1.96)$

$0.89(0.63-1.26) \quad 0.98(0.68-1.40)$ 


\section{Current weight perception}

Just about right

Overweight

Underweight

\section{Desired Weight}

Stay the same

Weigh more

1.00

1.00

1.00

1.00

1.00

1.00

$1.67(1.06-2.61) \quad 0.80(0.49-1.30) \quad 0.85(0.52-1.38) \quad 1.46(1.02-2.09) \quad 1.11(0.75-1.64) \quad 1.06(0.71-1.60)$

$1.41(1.15-1.72) \quad 1.15(0.91-1.44) \quad 1.04(0.79-1.37) \quad 0.90(0.66-1.23) \quad 0.87(0.62-1.23) \quad 0.94(0.67-1.30)$

${ }^{1}$ Clinically relevant depression: Patient Health Questionnaire (PHQ) score $\geq 10$

${ }^{2}$ Model 1 adjusted for race (Non-Hispanic White, Non-Hispanic Black, Mexican American and Other) and age at study entry. Model 2 additionally adjusted for smoking (never, former, current smoker), education (High school or less, Some college or more, Unknown), marital status (Never married/Widowed,

Divorced/Separated, Married/Living as married, Unknown), physical activity (Moderate and/or vigorous, Inactive), alcohol consumption (Non-drinker, Moderate drinker, Binge drinker, Heavy drinker) and self-rated health status (Excellent, Very good, Good, Fair, Poor, Don't know). Model 3 further adjusted for measured BMI $\left(\mathrm{kg} / \mathrm{m}^{2}\right.$, continuous)

${ }^{3}$ BMI: Body Mass Index 


\section{References}

Agrawal, P., Mercer, A., Hassanali, J., Carmack, C., Doss, D., Murillo, R., 2018. Gender Differences in the Association Between Alcohol Use and Sedentary Behavior Among Adults. American journal of health promotion : AJHP 32, 1576-1581.

Al Mamun, A., Cramb, S., McDermott, B.M., O'Callaghan, M., Najman, J.M., Williams, G.M., 2007. Adolescents' perceived weight associated with depression in young adulthood: a longitudinal study. Obesity (Silver Spring, Md.) 15, 3097-3105.

Ali, M.M., Fang, H., Rizzo, J.A., 2010. Body weight, self-perception and mental health outcomes among adolescents. The journal of mental health policy and economics 13, 53-63.

Borrud, L., Chiappa, M.M., Burt, V.L., Gahche, J., Zipf, G., Johnson, C.L., Dohrmann, S.M., 2014. National Health and Nutrition Examination Survey: national youth fitness survey plan, operations, and analysis, 2012. Vital and health statistics. Series 2, Data evaluation and methods research, 1-24.

Cash, T.F., 2004. Body image : a handbook of theory, research and clinical practice. Guilford Press, New York; London.

Cash, T.F.P., Thomas., 1990. Body images: Development, deviance, and change. Guilford Press, New York, NY, US.

de Wit, L.M., van Straten, A., van Herten, M., Penninx, B.W., Cuijpers, P., 2009. Depression and body mass index, a u-shaped association. BMC public health 9, 14.

Deeks, A.A., 2004. Is this menopause? Women in midlife--psychosocial issues. Australian family physician 33, 889-893.

Dittmar, H., Howard, S., 2004. Thin-Ideal Internalization and Social Comparison Tendency as Moderators of Media Models' Impact on Women's Body-Focused Anxiety. Journal of Social and Clinical Psychology 23, 768-791.

Evans, E.M., Rowe, D.A., Racette, S.B., Ross, K.M., McAuley, E., 2006. Is the current BMI obesity classification appropriate for black and white postmenopausal women? International journal of obesity (2005) 30, 837-843.

Frederick, D.A., Buchanan, G.M., Sadehgi-Azar, L., Peplau, L.A., Haselton, M.G., Berezovskaya, A., Lipinski, R.E., 2007. Desiring the muscular ideal: Men's body satisfaction in the United States, Ukraine, and Ghana. Psychology of Men \& Masculinity 8, 103-117.

Friedman, K.E., Reichmann, S.K., Costanzo, P.R., Musante, G.J., 2002. Body image partially mediates the relationship between obesity and psychological distress. Obesity research 10, 33-41. Gaskin, J.L., Pulver, A.J., Branch, K., Kabore, A., James, T., Zhang, J., 2013. Perception or reality of body weight: Which matters to the depressive symptoms. Journal of Affective Disorders 150, 350-355. Goldfarb-Rumyantzev, A.S., Rout, P., Sandhu, G.S., Khattak, M., Tang, H., Barenbaum, A., 2010. Association between social adaptability index and survival of patients with chronic kidney disease. Nephrology Dialysis Transplantation 25, 3672-3681.

Gray, W.N., Crawford, M.J., Follansbee-Junger, K., Dumont-Driscoll, M.C., Janicke, D.M., 2012. Associations between actual and perceived weight and psychosocial functioning in children: the importance of child perceptions. Childhood obesity (Print) 8, 147-154.

Halliwell, E., Dittmar, H.J.S.R., 2003. A Qualitative Investigation of Women's and Men's Body Image Concerns and Their Attitudes Toward Aging. 49, 675-684.

Holsen, I., Kraft, P., Roysamb, E., 2001. The Relationship between Body Image and Depressed Mood in Adolescence: A 5-year Longitudinal Panel Study. Journal of health psychology 6, 613-627.

Hong, G.W., Hong, S.M., 2019. Relationships among Body Mass Index, Body Image, and Depression in Korean Adults: Korea National Health and Nutrition Examination Survey 2014 and 2016. J Obes Metab Syndr 28, 61-68.

Jackson, K.L., Janssen, I., Appelhans, B.M., Kazlauskaite, R., Karavolos, K., Dugan, S.A., Avery, E.A., Shipp-Johnson, K.J., Powell, L.H., Kravitz, H.M., 2014. Body image satisfaction and depression in midlife women: the Study of Women's Health Across the Nation (SWAN). Archives of women's mental health 17, 177-187.

Kamody, R.C., Thurston, I.B., Decker, K.M., Kaufman, C.C., Sonneville, K.R., Richmond, T.K., 2018. Relating shape/weight based self-esteem, depression, and anxiety with weight and perceived physical health among young adults. Body Image 25, 168-176.

Kim, Y., Austin, S.B., Subramanian, S.V., Kawachi, I., 2018. Body weight perception, disordered weight control behaviors, and depressive symptoms among Korean adults: The Korea National Health and Nutrition Examination Survey 2014. PLoS One 13, e0198841-e0198841.

Kroenke, K., Spitzer, R.L., Williams, J.B., 2001. The PHQ-9: validity of a brief depression severity measure. J Gen Intern Med 16, 606-613.

LaPierre, T.A., 2009. Marital Status and Depressive Symptoms Over Time: Age and Gender Variations. 58, 404-416. 
Lim, Y., Kim, B., 2017. Body Mass Index, Body Weight Perception, and Depressed Mood in Korean Adolescents. Journal of the Korean Academy of Child and Adolescent Psychiatry 28, 31-37.

Loprinzi, P.D., Loenneke, J.P., 2018. Leukocyte telomere length and mortality among U.S. adults:

Effect modification by physical activity behaviour. Journal of sports sciences 36, 213-219.

Luppino, F.S., de Wit, L.M., Bouvy, P.F., Stijnen, T., Cuijpers, P., Penninx, B.W., Zitman, F.G., 2010.

Overweight, obesity, and depression: a systematic review and meta-analysis of longitudinal studies.

Archives of general psychiatry 67, 220-229.

Mannan, M., Mamun, A., Doi, S., Clavarino, A., 2016. Is there a bi-directional relationship between depression and obesity among adult men and women? Systematic review and bias-adjusted meta analysis. Asian journal of psychiatry 21, 51-66.

Mintem, G.C., Gigante, D.P., Horta, B.L., 2015. Change in body weight and body image in young adults: a longitudinal study. BMC public health 15, 222.

Moore, M.T., Fresco, D.M., 2012. Depressive realism: A meta-analytic review. Clinical Psychology Review 32, 496-509.

Moussavi, S., Chatterji, S., Verdes, E., Tandon, A., Patel, V., Ustun, B., 2007. Depression, chronic diseases, and decrements in health: results from the World Health Surveys. Lancet (London, England) 370, 851-858.

Nguyen, H.A., Anderson, C.A.M., Miracle, C.M., Rifkin, D.E., 2017. The Association between Depression, Perceived Health Status, and Quality of Life among Individuals with Chronic Kidney Disease: An Analysis of the National Health and Nutrition Examination Survey 2011-2012. Nephron 136, 127-135.

Paans, N.P.G., Bot, M., Brouwer, I.A., Visser, M., Penninx, B., 2018. Contributions of depression and body mass index to body image. J Psychiatr Res 103, 18-25.

Richard, A., Rohrmann, S., Lohse, T., Eichholzer, M., 2016. Is body weight dissatisfaction a predictor of depression independent of body mass index, sex and age? Results of a cross-sectional study. BMC public health 16, 863-863.

Roberts, R.E., Duong, H.T., 2013. Perceived weight, not obesity, increases risk for major depression among adolescents. J Psychiatr Res 47, 1110-1117.

Rodgers, R.F., McLean, S.A., Paxton, S.J., 2015. Longitudinal relationships among internalization of the media ideal, peer social comparison, and body dissatisfaction: implications for the tripartite influence model. Developmental psychology 51, 706-713.

Rucker III, C.E., Cash, T.F., 1992. Body images, body-size perceptions, and eating behaviors among African-American and white college women. 12, 291-299.

Silva, D., Ferriani, L., Viana, M.C., 2019. Depression, anthropometric parameters, and body image in adults: a systematic review \%J Revista da Associação Médica Brasileira. 65, 731-738.

Slade, P.D., 1994. What is body image? Behaviour Research and Therapy 32, 497-502.

Smolak, L., 2004. Body image in children and adolescents: where do we go from here? Body Image 1, 15-28.

Sowers, M., Zheng, H., Tomey, K., Karvonen-Gutierrez, C., Jannausch, M., Li, X., Yosef, M., Symons, J., 2007. Changes in body composition in women over six years at midlife: ovarian and chronological aging. The Journal of clinical endocrinology and metabolism 92, 895-901.

Spitzer, R., Williams, J., Kroenke, K.J.R.C.B., 2014. Test Review: Patient Health Questionnaire-9 (PHQ-9). 57, 246-248.

Takacs, J., 2014. [Regular physical activity and mental health. The role of exercise in the prevention of, and intervention in depressive disorders]. Psychiatria Hungarica : A Magyar Pszichiatriai Tarsasag tudomanyos folyoirata 29, 386-397.

Tang, J., Yu, Y., Du, Y., Ma, Y., Zhu, H., Liu, Z., 2010. Association between actual weight status, perceived weight and depressive, anxious symptoms in Chinese adolescents: a cross-sectional study. BMC public health 10, 594 .

Thurston, I.B., Sonneville, K.R., Milliren, C.E., Kamody, R.C., Gooding, H.C., Richmond, T.K., 2017. Cross-sectional and Prospective Examination of Weight Misperception and Depressive Symptoms Among Youth with Overweight and Obesity. Prevention science : the official journal of the Society for Prevention Research 18, 152-163.

Vásquez, E., Batsis, J.A., Germain, C.M., Shaw, B.A., 2014. Impact of Obesity and Physical Activity on Functional Outcomes in the Elderly: Data From NHANES 2005-2010. Journal of Aging and Health 26, 1032-1046.

World Health Organisation, 2019. Depression,

http://www.who.int/mediacentre/factsheets/fs369/en/index.html.

World Health Organization, 2000. Obesity: preventing and managing the global epidemic. 
Zipf, G., Chiappa, M., Porter, K.S., Ostchega, Y., Lewis, B.G., Dostal, J., 2013. National health and nutrition examination survey: plan and operations, 1999-2010. Vital and health statistics. Ser. 1, Programs and collection procedures, 1-37. 